\title{
ABO Blood Groups and Hyperbaric Oxygen Therapy Effectiveness in Sudden Hearing Loss
}

\author{
Esin Eren ${ }^{1,3}$, Furkan Yıldırım², Özlem Giray ${ }^{1}$, Necat Y1lmaz ${ }^{1^{*}}$ \\ ${ }^{1}$ University of Health Science, Antalya Education and Research Hospital Central LC-MS/MS \\ Toxicology Laboratories \\ ${ }^{2}$ University of Health Science Antalya Education and Research Hospital Underwater and \\ Hyperbaric Medicine \\ ${ }^{3}$ Antalya Bilim University Health Vocational School
}

\begin{abstract}
The efficiency of hyperbaric oxygen (HBO) treatment according to ABO blood group system was evaluated in patients with idiopathic sudden sensorineural hearing loss (ISSNHL). Serum lipid values and novel atherogenic risk calculations of 346 patients who came to the clinic for HBO treatment were performed. HBO treatment efficiency was evaluated on ABO blood groups. The effect of serum lipid levels and atherogenic risk levels on HBO treatment was measured according to the blood groups of ISSNHL patients. The most important finding is that ISSNHL patients with $O$ blood group antigen benefit the least from HBO therapy. Moreover, patients with $O$ blood group antigen had statistically lower serum lipid values and atrogenic risk index than patients with non-O blood group. In addition, the frequency of $O$ blood group in ISSNHL patients was higher than in non-O blood groups. The effect of serum lipid values and atherogenic risk indices in ISSNHL patients receiving HBO therapy may vary according to ABO blood groups. The relationship between hyperbaric oxygen therapy and the ABO blood grouping system should be supported by further research.
\end{abstract}

Key words: Hyperbaric oxygen therapy, Atherogenic index, Atherogenic plasma index,

Lipoprotein combine index, Blood groups, Non-O blood group.

\section{Introduction:}

Human ABO blood type is determined by a gene on chromosome 9.Moreover, ABO blood group is the first genetic polymorphism recognized in humans (1). The ABO histocompatibility-expression of blood group antigens on the surface of erythrocytes and it is also expressed on the surface of platelets, endothelial and epithelial cells. Therefore, there is a large literature on the relationships between ABO blood group and different pathologies (1-3).

ISSNHL, in which HBO therapy is used in the treatment, is a common otological emergency with still uncertain cause

\footnotetext{
* Corresponding Author: Necat Yılmaz, Department of Clinical Chemistry and LC/MS-MS Laboratory, Antalya Training and Research Hospital, Antalya, Turkey. E-mail: necatyilmaz@ hotmail.com. Tel: +90 357 8305. ORCID ID: 0000-0002-3865-9156.
} 
(4).The $\mathrm{ABO}$ blood group, which has a role in the pathophysiology of a wide range of diseases in the literature, may also include ISSNHL (5).Because there are studies showing that there is a relationship between $\mathrm{ABO}$ blood groups and hearing loss. For example, according to studies, individuals with $\mathrm{O}$ blood group have the lowest amplitude in both ears (6-8). People with blood type $\mathrm{O}$ show a higher prevalence of hearing loss after exposure to industrial noise. However, it is not clear whether the blood group status affects the auditory brainstem responses reflecting cochlear hair cell and auditory nerve bioelectric activity (6-8).In addition, among the various factors reported to affect the susceptibility to hearing loss, race, geographical region, eating habits, dyslipidemia, cholesterol, etc. factors can be included (9). Therefore, the importance of blood lipids in the pathogenesis of ISSNHL has been widely reported in the literature (9-11). In fact, high levels of low-density lipoprotein cholesterol (LDL), total cholesterol (TC), and apolipoprotein B (Apo-B) have been suggested as risk factors for ISSNHL pathology (7-10). Consequently, the importance of blood lipids in the pathogenesis of ISSNHL has been widely discussed in the literature (9). But, the role of atherogenic risk factors in the pathogenesis of ISSNHL is still controversial $(5,9)$.

A clinical study showed that TC, LDL-C, and Apo-B levels were significantly higher in patients with ISSNHL compared to control subjects (11). Also it has been reported that ISSNHL is associated with many vascular and coagulation diseases (35)

Dyslipidemia is one of the most important cardiovascular risk factors, which is also defined as a cardiovascular risk factor in
European societies and evidence has shown that there is a relationship between ISSNHL and dyslipidemia $(6,7)$. These findings led to the hypothesis that non-O blood group antigens may have higher levels of dyslipidemia and an inflammatory condition and oxidative stress may occur (3-5). Inflammation and oxidative stress may play a critical role in ISSNHL disease $(11,12)$. Because impaired cochlear perfusion appears to be an important etiopathogenetic event in ISSNHL. The cochlea is an end organ that is metabolically dependent on a supply of nutrients and oxygen to maintain its normal physiological function. It is very sensitive to changes in blood circulation. Early histopathological studies showed that sclerotic changes in the blood vessels of older ears contribute to inner ear degeneration and other cochlear changes (11-13). Therefore, the effect of the ABO blood system on atherosclerosis susceptibility is thought to be mediated in part by its changed on blood lipid levels. Today, it is well known that atherogenic risk calculation plays an important role in clinical practice. However, despite their obvious clinical significance, the computational risk of atherogenicity of ABO blood group antigens is poorly known (1, 9). However possible relationship of blood lipids levels to blood groups may be one of the causes of ISSNHL.

However, there is no study in the literature showing the distribution of atherogenic indices in ISSNHL according to blood groups. In addition, the contribution of ABO blood group distribution to the response efficiency of $\mathrm{HBO}$ therapy in ISSNHL patients is unknown. The aim of this preliminary study is to evaluate the possible effect of the distribution of new 


\section{E. Eren et al.}

atherogenic risk indices by blood groups in ISSNHL patients on HBO therapy.

\section{Materials and Methods}

\section{Subjects}

Patients known according to $\mathrm{ABO}$ blood type laboratory records and ISSNHL patients who came to hyperbaric clinic for treatment were included in the study. Those selected for inclusion in this study are adults over the age of 18 who have been informed about the study and have voluntary consent. The age range of the working group formed is 18-58. A total of 346 patients affected by ISSNHL were included in the study. Exclusion criteria for patients with ISSNHL were: acute inflammation, infection, autoimmune disorders, cochlear-vestibular dysfunction, otologic surgery, neurological disorders, ototoxic drug use, neoplasm, or other chronic diseases. In addition, those who used drugs due to lipid metabolism disorder and those with features suggestive of diabetes and metabolic syndrome were also exclusion criteria. Body mass index, waist circumference, systolic blood pressure, and diastolic blood pressure measurements were performed by physicians in all patients at rest in the hyperbaric clinic. In addition, data on clinical and demographic characteristics such as age, chronic disease history, and smoking status were collected from medical records. Post-treatment improvement of hearing results were analyzed according to ISSNHL criteria. All subjects included gave their informed consent to participate in the study. The study was approved by the ethics committee of the relevant institute.

\section{HBO therapy}

HBO treatment was applied as follows in patients with ISSNHL. Each consecutive
HBO treatment session was administered five days a week, each session was 120 minutes. Clinical follow-up of all patients in the hyperbaric clinic was performed and a multi-atmospheric hyperbaric chamber (Baroks, MUL35) was used. During HBO therapy, each patient breathes $100 \%$ oxygen at 2.5 at high atmospheric pressure. Also, all sessions of HBO had five-minute air break times every 30 minutes. In each HBO treatment session, patients were also given 15 minutes of compression and 10 minutes of opening time.

\section{Samples}

Venous blood samples were collected from patients and control subjects after at least $12 \mathrm{~h}$ of fasting for the evaluation of serum lipids. The samples were collected into noadditive vacutainer tubes. Blood samples were centrifuged, and serum was transferred to laboratory until further analysis.

\section{Lipid parameters}

Auto-analyzer Beckman AU5800® (Beckman Coulter Diagnostics, CA, USA) for the measurement of, TC, low density lipoprotein cholesterol (LDL-C), high density lipoprotein cholesterol (HDL-C) and triglyceride (TG) were used in the study and commercial diagnostic reagent kits of the same brand were used. Estimation of serum very low density lipoprotein (VLDL) and non-HDL-C concentrations were calculated using the formula as follows: [VLDL cholesterol] $(\mathrm{mmol} / \mathrm{L})=[$ Triglyceride $] / 2.2$ and nonHDL-C $=(\mathrm{TC})-(\mathrm{HDL}) . \mathrm{TC}, \mathrm{LDL}-\mathrm{C}$, and TG were measured by enzymatic methods and HDL-C by a direct method. TC, TG, HDL-C and LDL-C concentrations were double measured in all subjects.

Non-traditional atherogenic indices Especially in this study, the atherogenic index (AI), atherogenic plasma index 
(AIP), lipoprotein combine index (LPCI) were calculated from the values of routine lipid parameters $(\mathrm{mmol} / \mathrm{L})$. In fact, AI is defined as the ratio of non-HDL-C to HDL-C (non-HDL-C / HDL-C); LCI is defined as the ratio of TC $\times$ TG $\times$ LDL to HDL-C.

AIP was calculated as a logarithm of the ratio of the molar concentration $(\mathrm{mmol} / \mathrm{l})$ of TG to HDL-C (i.e., log [TG/HDL-C]) [29].

Simply atherogenic indices were calculated using the following formulas: $\mathrm{AI}$; nonHDL-C / HDL-C, LCI; TC x TG x LDL / HDL-C. Unlike these new index calculations, logarithm values of base 10 were used in the calculation of AIP.AIP; $\log 10$ (TG / HDL-C). It was calculated according to the ratio of logarithmic TG concentration in the AIP to logarithmic HDL-C. AIP is an index that has been classified for atherosclerosis in the literature: Low risk of AIP $<0.1, \quad 0.1$ to 0.24 for medium risk and more than 0.24 for high risk.

\section{Statistical Analyses}

All continuous variables are presented as the median and interquartile range (25th and 75th percentiles) and compared using the Mann-Whitney test. Categorical data are shown as percentages and compared using the Chi square test. The statistical analysis was performed using MedCalc ${ }^{\circledR}$ software. A value of $p<0.05$ was considered statistically significant.

\section{Results}

The mean age of ISSNHL patients and the number of female patients (n: 204) of 346 patients included in this study are given in table 1. Other demographic and clinical data of the patients are given in Table 1. In addition, the distributions of blood group antigens of all ISSNHL patients are given in Table 1. While the majority of patients had $\mathrm{O}$ (n: 154) blood type, ISSNHL patients with $\mathrm{AB}$ blood group had a statistically higher smoking percentage than other blood group patients. In addition, serum uric acid (UA) level was statistically higher in patients with blood type A, but UA and fasting blood glucose (FBG) levels were within normal limits in all blood groups (Table 1).

Table 1: Characteristics of the ISSNHL patients.

\begin{tabular}{|c|c|c|c|c|}
\hline \multirow{2}{*}{$\begin{array}{l}\text { Baseline } \\
\text { characteristics }\end{array}$} & A (n:133) & B (n:32) & AB (n:27) & 0 (n:154) \\
\hline & \multicolumn{4}{|c|}{ Mean \pm SD } \\
\hline Age(years) & $44.9 \pm 11$ & $43.9 \pm 8.4$ & $47.03 \pm 8$ & $44.05 \pm 10$ \\
\hline BMI $\left(\left(\mathrm{kg} / \mathbf{~ m}^{2}\right)\right.$ & $26.3 \pm 3$ & $25.8 \pm 2.9$ & $26.8 \pm 2.61$ & $25.4 \pm 2.77$ \\
\hline Smoking(\%) & 45 & 38 & $47 *$ & 21 \\
\hline HT(\%) & 7.2 & 7.8 & 8.2 & 7.9 \\
\hline FBG(mg/dl) & $101 \pm 11$ & $98 \pm 9$ & $103 \pm 12$ & $100 \pm 13$ \\
\hline $\mathrm{UA}(\mathrm{mg} / \mathrm{dl})$ & $5.8 \pm 1.3^{*}$ & $5.5 \pm 1.4$ & $5.5 \pm 1.46$ & $5.36 \pm 1.24$ \\
\hline
\end{tabular}

The serum lipid values of the patients included in the study and the atherogenic risk indexes calculated from these lipid values are given in table 2. Also lipid values of ISSNHL patients are given in table 2 .
The important finding is that the AIP and LPCI are statistically significantly lower in individuals with $\mathrm{O}$ blood group than other blood groups. Also, TG and TG / HDL-C ratio are lower in patients with $\mathrm{O}$ blood group. However, in patients with AB blood 


\section{E. Eren et al.}

group, TC and AI risk index were significantly higher than other blood groups (Table 2).

In addition, the response of ISSNHL patients to HBO therapy differed significantly compared to blood group antigens. Perhaps an important finding that draws attention is that patients with $\mathrm{O}$ blood group antigen had the lowest response to HBO therapy despite low atherogenic risk values. Unfortunately, only $16 \%$ of patients with blood type $\mathrm{O}$ antigen showed complete recovery with $\mathrm{HBO}$ therapy.

Table 2: Lipid parameters of ISSHL Patients.

\begin{tabular}{|c|c|c|c|c|}
\hline \multirow[t]{2}{*}{ Overall lipid variables(n:345) } & \multicolumn{4}{|c|}{ ABO Blood Groups } \\
\hline & A (n:133) & B (n:32) & AB (n:27) & $O(n: 153)$ \\
\hline \multicolumn{5}{|c|}{ Traditional Lipid Parameters } \\
\hline TC(mmol/L) & 5.6 & 5.1 & $5.8^{*}$ & 5.18 \\
\hline TG(mmol/L) & 1.76 & 2.27 & 1.79 & $1.44 * *$ \\
\hline LDL-C(mmol/L) & 3.54 & 3.23 & 3.62 & 3.28 \\
\hline HDL-C(mmol/L) & 1.47 & 1.57 & $1.24 *$ & 1.45 \\
\hline VLDL(mmol/L) & 0.35 & 0.59 & 0.36 & $0.11 * * *$ \\
\hline \multicolumn{5}{|c|}{ Novel Lipid Indices } \\
\hline API & 0.18 & 0.17 & 0.20 & $0.15^{* *}$ \\
\hline $\mathbf{A I}$ & 2.87 & 2.24 & $3.5^{*}$ & 2.9 \\
\hline LPCI & 24.1 & 23.8 & 24.8 & $20.4 * *$ \\
\hline TG/HDL-C & 1.19 & 1.44 & 1.41 & $0.99 * *$ \\
\hline
\end{tabular}

On the contrary, the rate of those who did not respond to HBO treatment was $30 \%$. Fortunately, $54 \%$ of patients improved after HBO therapy (Table 3).

However, the number of patients recovered the most was in ISSNHL patients with B blood group antigen (31\%), followed by AB (27\%) and A (18.3) blood groups, respectively. Also, $\mathrm{AB}$ blood group had the lowest rate of response to failed treatment with HBO therapy in ISSNHL patients (Table 3).

Table 3: Patients recovering with HBO.

\begin{tabular}{lccccccccc}
\hline $\begin{array}{l}\text { ABO Blood } \\
\text { Groups }\end{array}$ & $\mathbf{0 -}$ & $\mathbf{0 +}$ & $\mathbf{A -}$ & $\mathbf{A +}$ & $\mathbf{A B}-$ & $\mathbf{A B +}$ & $\mathbf{B}-$ & $\mathbf{B}+$ & $\mathbf{n}$ \\
\hline As it is & 2 & $48(30 \%)$ & 4 & $24(20 \%)$ & & $5(22 \%)$ & $1(0.03 \%)$ & 84 \\
\hline Improvement & 4 & $76(54 \%)$ & 9 & $74(69.7 \%)$ & 4 & $12(72 \%)$ & 3 & $19(75.8 \%)$ & 201 \\
\hline Healing & & $24(16 \%)$ & 1 & $21(18.3 \%)$ & 1 & $5(27 \%)$ & $9(31 \%)$ & 61 \\
\hline n: & 6 & 148 & 14 & 119 & 5 & 22 & 3 & 29 & 346
\end{tabular}

\section{Discussion}

Previous studies have reported several atherogenic risk factors for ISSNHL. Among these risk factors, the rates of diabetes mellitus and hypercholesteremia are higher in patients with ISSNHL compared to the control group $(14,15)$. In addition, studies have also shown that patients with ISSNHL have a higher risk of stroke $(14,15)$.Indeed according to the results of this study, atherogenic risk factors and blood group data may be cost effective in determining the efficacy of HBO therapy.

In this preliminary study, we first showed the lower efficacy of ISSNHL patients 
with blood group antigen $\mathrm{O}$ in the treatment HBO. Also this study has showed that more patients of ISSNHL who applied for $\mathrm{HBO}$ treatment had $\mathrm{O}$ blood group antigen.

We also show that the distribution of atherogenic risk indexes is not associated with the risk of ISSNHL. Because the ISSNHL patients included in this study did not have a high atherogenic risk index.

Relationships between hearing and blood lipid levels have long been the focus of scientific research. Patients with ISSNHL often have hyperlipidemia. The cochlea is an end organ that is metabolically dependent on a supply of nutrients and oxygen to maintain its normal physiological function. It is very sensitive to changes in blood circulation. Cochlear ischemia is considered one of the most important causes of ISSNHL. Hyperlipidemia may contribute to cochlear ischemia due to increased blood viscosity. Increased blood viscosity can reduce inner ear blood flow and cause inner ear damage [17]. Moreover, lipid metabolic disorders can lead to lipid accumulation in cochlear hair cells and damage to cochlear nerve cells, followed by inhibition of neural transduction (17, 18). Moreover, hearing loss due to hyperlipidemia tends to improve with diet control and antilipidemic therapy (19).

However, the findings obtained in this study showed that the $\mathrm{O}$ blood group ISSNHL patients with normal TC and TG values gave the lowest response to HBO treatment. On the contrary, patients with $\mathrm{AB}$ and $\mathrm{A}$ blood groups gave a higher response to $\mathrm{HBO}$ treatment compared to $\mathrm{O}$ blood group, although they had TC and TG levels above normal values. In addition individuals with $\mathrm{B}$ blood group gave the best response to HBO treatment despite high borderline TG levels. Yet there is no study showing the relationship of blood group antigens with $\mathrm{HBO}$ treatment in ISSNHL patients. The findings of this preliminary study need to be supported by further studies. The finding that might be suggested from this study is that ISSNHL patients with $\mathrm{O}$ blood group antigen are less likely to be injured than HBO therapy. The negative might be effects of $\mathrm{O}$ blood antigens on microcirculation may lies in its affecting the rheological properties of blood, the composition of membrane lipids, protein-lipid interactions in membrane structures and the activities of membrane-bound enzymes. Recently, researchers reported that individuals with blood group $\mathrm{O}$ are relatively more susceptible to noise-induced hearing loss (20). To confirm ABO blood group hypothesis, measuring ultrahigh-frequency auditory sensitivity in individuals with different blood groups would be beneficial Also the reduction in amplitude of ultrahigh-frequency in blood group $\mathrm{O}$ indicates a possible reduction in outer hair cell function. However, HBO therapy is generally very effective on recovery from ISSNHL patients. In patients with ISSNHL blood group $\mathrm{O}$, the lipid profile indicates a statistically lower atherogenicity. As a result, the novel atherogenic indexes AIP and AI index are lower in patients with blood group $\mathrm{O}$ than non-O blood groups. To date, atherogenic indices by blood grouping have not been studied in ISSNHL patients. The results of the present study are in consensus with reports by previous studies who also reported reduced HBO treatment response in individuals with blood group $\mathrm{O}(20,21)$.

\section{Conclusion}

This study attempted to determine effects 
of blood group type on ISSNHL using atherogenic indices and lipid measurements during HBO therapy. The results of the study showed a significant reduction in good response to $\mathrm{HBO}$ therapy in individuals with blood type $\mathrm{O}$ compared to individuals with other blood groups. However, there was no significant difference in response to HBO treatment between non-O blood groups. Therefore, it can be considered that ISSNHL patients with blood group $\mathrm{O}$ antigen may be genetically susceptible to ISSNHL and their lipid levels are not effective. Further studies on larger individual groups are required to generalize the results.

\section{Conflict of Interest}

The authors declare that they have no conflict of interest.

\section{Acknowledgment}

No institution has given financial support to the study. All researchers contributed equally to the study.

\section{References:}

1. Eren E, Yılmaz N, Koçtekin B, et al. Highdensity lipoprotein functionality and $\mathrm{AB}$ blood phenotype. Iraqi J Hematol 2020;9:66-71. DOI: 10.4103/ijh.ijh_10_20.

2. Yang Y, Hood LJ, McPherson B. Association between $\mathrm{ABO}$ blood group status and cochlear/neural function: auditory brainstem response findings. Acta Otolaryngol. 2021 Jan 11:1-6. doi: 10.1080/00016489.2020.1858236.

3. Birinci, S, Koçtekin, B, Eren E, et al. Lecithin- cholesterol acyltransferase and relationship with Platelet-activating factor in $\mathrm{AB}$ blood phenotype. Bali Medical Journal 2020 9(1): 332-338. DOI: 10.15562/bmj.v9i1.1746.

4. Rhee TM, Hwang D, Lee JS, et al. Addition of Hyperbaric Oxygen Therapy vs Medical Therapy Alone for Idiopathic Sudden Sensorineural Hearing Loss: A Systematic Review and Meta-analysis. JAMA Otolaryngol Head Neck
Surg. 2018 Dec 1;144(12):1153-1161. doi: 10.1001/jamaoto.2018.2133.

5. Eren E, Yıldırım F, Giray O, et al. Hyperbaric Oxygen Treatment is Associated with Lipid Inflammatory Response Assessed Using Serum Platelet Activating Factor, Polish Hyperbaric Research, 67(2), 103-108. doi: 10.2478/phr-20190009.

6. Henderson D, Subramaniam M, Boettcher FA. Individual susceptibility to noise-induced hearing loss: an old topic revisited. Ear Hear. 1993 Jun; 14(3): 152-68. doi: 10.1097/00003446199306000-00002. PMID: 8344472.

7. Quaranta N, Squeo V, Sangineto M, et al. High Total Cholesterol in Peripheral Blood Correlates with Poorer Hearing Recovery in Idiopathic Sudden Sensorineural Hearing Loss. PLoS One. 2015 Jul 24; 10(7):e0133300. doi: 10.1371/journal.pone.0133300.

8. Byl FM Jr. Sudden hearing loss: eight years' experience and suggested prognostic table. Laryngoscope. 1984 May; 94(5 Pt 1):647-61.

9. Kaneva AM, Yanov YK, Bojko SG, et al. The atherogenic index (ATH index) as a potential predictive marker of idiopathic sudden sensorineural hearing loss: a case control study. Lipids Health Dis. 2019 Mar 15; 18(1):64. doi: 10.1186/s12944-019-1016-5.

10. Weng $\mathrm{T}$, Devine EE, $\mathrm{Xu} \mathrm{H}$, et al. A clinical study of serum lipid disturbance in Chinese patients with sudden deafness. Lipids Health Dis. 2013 Jul 3; 12:95. doi: 10.1186/1476-511X-12-95.

11. Suzuki K, Kaneko M, Murai K. Influence of serum lipids on auditory function. Laryngoscope. 2000 Oct; 110 (10 Pt 1): 1736-8. doi: 10.1097/00005537-200010000-00033.

12. Cadoni G, Scorpecci A, Cianfrone F, et al. Serum fatty acids and cardiovascular risk factors in sudden sensorineural hearing loss: a case-control study. Ann Otol Rhinol Laryngol. 2010 Feb; 119(2):82-8. doi: 0.1177/000348941011900203.

13. Capaccio P, Pignataro L, Gaini LM, et al. Unbalanced oxidative status in idiopathic sudden sensorineural hearing loss. Eur Arch Otorhinolaryngol. 2012 Feb; 269(2):449-53. doi: 10.1007/s00405-011-1671-2.

14. Furuhashi A, Matsuda K, Asahi K, et al. Sudden deafness: long-term follow-up and recurrence. Clin Otolaryngol Allied Sci. 2002 Dec; 27(6):458-63. doi: 10.1046/j.13652273.2002.00612.x.

15. Jung SY, Shim HS, Hah YM, et al. Association of Metabolic Syndrome With Sudden Sensorineural Hearing Loss. JAMA Otolaryngol Head Neck Surg. 2018;1;144(4):308-314. doi: 10.1001/jamaoto.2017.3144.

16. Lee JS, Kim DH, Lee HJ, et al. Lipid profiles and obesity as potential risk factors of sudden sensorineural hearingloss. PLoS One. 2015; doi:10:e0122496. 


\section{E. Eren et al.}

17. Fischer ME, Schubert CR, Nondahl DM, et al. Subclinical atherosclerosis and increased risk of hearing impairment. Atherosclerosis. 2015 Feb; 238(2): 344-9. doi: 10.1016/j.atherosclerosis.2014.12.031.

18. Shi X. Cochlear Vascular Pathology and Hearing Loss. In: Ramkumar V., Rybak L. (eds) Inflammatory Mechanisms in Mediating Hearing Loss. Springer, Cham.2018, doi: 10.1007/978-3319-92507-3_4.

19. Mohammed AA. Lipid Profile among Patients with Sudden Sensorineural Hearing Loss. Indian J Otolaryngol Head Neck Surg. 2014 Dec; 66(4):425-8. doi: 10.1007/s12070-014-0744-0.

20. Chow KT, McPherson B, Fuente A. Otoacoustic emissions in young adults: Effects of blood group. Hear Res. 2016 Mar; 333:194-200. doi: 10.1016/j.heares.2015.09.006.

21. Prabhu P, Chandrashekhar A, Cariappa J, et al. Effect of Blood Group on Ultrahigh Frequency Auditory Sensitivity. Int Arch Otorhinolaryngol. 2018; 22(4):364-367. doi: $10.1055 / \mathrm{s}-0037-1613711$. 